\title{
Genetics and genomics education: The next generation
}

\author{
Bruce R. Korf, $M D, P h D$
}

\begin{abstract}
$\mathrm{T}$ here is debate about many things regarding how genetics and genomics will be integrated into medicine, for example, the role of direct-to-consumer testing, the cost-effectiveness of pharmacogenetic testing, or the value of whole genome singlenucleotide polymorphism analysis in risk assessment. One point, however, that seems to be widely accepted is that current approaches to health professional education do not prepare providers for the new era of genomic medicine. As with "Moore's Law," which notes the rapid increase in the density of components that can be fit onto a computer chip, there is an explosive growth of information density required for the practice of medicine. The challenge is that neither curricula nor the students they are designed to teach have the seemingly limitless capacity to accommodate such a volume of new information. How are we to educate a health care workforce to make best use of the power of genetics and genomics over the coming generation? I would like to suggest some guiding principles that may be helpful in meeting the challenge.
\end{abstract}

\section{FOCUS ON COMPETENCIES}

Although I have not done a systematic survey, I would bet that most medical students know the elements of DNA structure and the processes of transcription and translation, yet few could explain to a patient the meaning of a "variant of unknown significance." Similarly, most could probably explain the difference between dominant and recessive inheritance, but few could obtain and interpret a multigenerational family history complete with real-world complexities such as nonpenetrance, misattributed paternity, and so on. The Liaison Committee on Medical Education requires that genetics be taught to medical students, and there are several lists of genetics concepts that might be included in the curriculum, yet little has been written on what we really expect physicians to be able to do using their knowledge, skills, and attitudes about genetics.

In the report "Scientific Foundations for Future Physicians," prepared by a committee sponsored jointly by the Howard Hughes Medical Institute and the Association of American Medical Colleges, the competency related to genetics and genomics is stated succinctly: "Use the principles of genetic transmission, molecular biology of the human genome, and population genetics to infer and calculate risk of disease, to institute an action plan to mitigate this risk, to obtain and interpret family history and ancestry data, to order genetic tests, to guide therapeutic decision-making, and to assess patient risk." This statement is followed by several learning objectives and examples. If this does not seem like much attention to a discipline that is one of the major engines of medical progress, realize that all other scientific disciplines important in medical

From the Department of Genetics, University of Alabama at Birmingham, Birmingham, Alabama.

Bruce R. Korf, MD, PhD, Department of Genetics, University of Alabama at Birmingham, Birmingham, AL. E-mail: bkorf@uab.edu.

Disclosure: The author declares no conflict of interest

Published online ahead of print January 28, 2011.

DOI: $10.1097 /$ GIM.0b013e31820986cd education get similar terse treatment. That is because it is the job of faculty to fill in the blanks and build a curriculum to use genetics and genomics in their practice or research. I urge our community to take the lead here by defining not what the future physician should know, but what he or she should be able to do. The same can be said for other areas in the health professions, such as nursing, physician assistants, and so on.

\section{DEVELOP AN INTEGRATED APPROACH TO EDUCATION IN GENOMIC MEDICINE}

Education about genetics and genomic medicine is not a task only for health professional education. Genomic medicine will touch every life and virtually every profession in the coming generation. Therefore, the effort to develop competencies must be broad-based. It should be obvious that health professional education would benefit from having a better-prepared group of matriculating students. This point was recognized in the Howard Hughes Medical Institute/Association of American Medical Colleges document, which also proposed competencies in the basic sciences for premedical students, and the need for reevaluation of the Medical College Admission Test and the US Medical Licensing Examination, both of which are now underway. Surely it would be better to be able to assume knowledge of transcription and translation, and then help medical students reach a new level of competency in application of concepts like genetic variation.

The need to prepare undergraduates who are not planning a health professional career, or graduate or professional students in engineering, law, and business about genetics and genomics may be less obvious but could also make a big difference. Should we expect a general undergraduate (or maybe a high school graduate) to be able to collect family history information from relatives or to make an informed decision about direct-toconsumer testing? Should a business student be able to assess the effect of pharmacogenetic testing on a pharmaceutical company business plan or on health insurance rates for small businesses? Should an attorney be trained to recognize the pitfalls in paternity testing or the basis for intellectual property law related to genetic testing or pharmaceutical development? The competency-based approach can and should be applied to any point in the educational continuum, resulting not only in better-prepared health professional students but also a more receptive society.

\section{DEVELOP POINT-OF-CARE TOOLS}

No matter how well educated our health professionals become, the density of information about genetics and genomics (not to mention most other areas of medicine) is rapidly exceeding human capacity to absorb and remember. It is said (I am not a pilot) that a modern jumbo jet could not be safely landed if all of the computer systems failed simultaneously. This is not to underestimate the importance of a skilled pilot, but the days of flying by instinct with the wind in one's hair are long gone in commercial (or military) aviation. The same is becoming true in medicine: there is too much to know, too much data to sort, for a health professional to keep everything in his or her head and 
provide state-of-the-art care. This does not mean that physicians should pay more attention to their computers and electronic health records than to listening to their patients. On the contrary; the pilot must constantly survey the environment, knowing that critical settings of engine or wing performance can be adjusted automatically. Medical decision support systems must be designed so that health professionals can rely on access to up-tothe-minute relevant information so that they can respond appropriately to the concerns that they hear upon interacting with their patients. Such well-designed and properly used systems can improve rather than degrade the human encounter that is the essence of the physician-patient interaction.

\section{GENETICS PROFESSIONALS MUST PREPARE FOR THE ERA OF GENOMIC MEDICINE}

It is conceivable that virtually all medical decisions will someday be informed at least, in part, by genetic analysis, yet it is inconceivable that a medical geneticist will be involved in every such decision. The wider use of genetic and genomic approaches is inevitable and should be celebrated as one of the triumphs of our era. Many genetic tests will lend themselves to relatively straightforward application and interpretation. As any medical geneticist knows, however, it does not take long to "step through the looking glass" into a world where straightforward rules do not seem to apply. This is exemplified by the need to interpret apparently silent mutations that disrupt splice enhancers, previously undescribed copy number variants, or the results of genetic testing for disorders of genomic imprinting. We can and should train our medical colleagues to recognize the power of genetics in medical practice and to interpret routine tests, but we must also be prepared to provide support when our colleagues and their patients venture into deeper, less wellcharted waters. This will demand much of the professional medical geneticist, who will need to be adept at stepping beyond familiar territories of dysmorphology, prenatal diagnosis, and biochemical genetics. It will necessitate an evolution of medical genetic training, including becoming adept at interpreting data derived from whole genome sequencing and assessing risk for common and rare disorders. It will also require increasing the numbers of other genetics professionals, especially genetic counselors.

I suspect that many medical geneticists were drawn to the field because it has always existed at the frontier of medicine. We may have paid a price for living on the edge in this way, in terms of not being at the center of power, for example, where reimbursement decisions are made. But there has also been a payoff, in terms of the excitement of watching the front of knowledge advance before our eyes and being in the position to rapidly translate that knowledge into better tools for diagnosis and treatment of our patients. We now have even larger challenges and rewards within our grasp, and we need to inspire a new generation of students to join us in this once-in-a-lifetime endeavor. 